\title{
Building Taiwan Aeropolis of Airports through Infrastructuring Care and Integrity
}

\author{
Li-Yen Hsu \\ Department of Architecture, China University of Science and Technology, Taipei 11581, Taiwan, R.O.C.
}

\begin{abstract}
Global changes, including the rise of the sea level and frequent terrible disasters, are apparently threatening the living environment. For environmental mitigation, security, and sustainability, Taiwan should have reliable, incorporative area-based security-information networks to protect their environments. Plural (dual) surveillance which is rooted in biologic senses, e.g., from two-eyes, can have the feature of fault-tolerance, availability and maintainability in monitoring tasks. Further, it can fit the contemporary wireless communication methods being incorporated with MIMO (multi-in multi-out), to prevent information loss, interference, unexpected changes caused by such as clogged water and chemical reactions. Consequently, network prototypes, including SW (spider-web)network and CCC (cube-connected cycle), are proactively suggested. More reliable capabilities, including those encouraging local ferries and drones, for efficient logistic operation and mixed-use buildings with quality assured performance, can be good for smart urban growth, resource utilization in Taiwan, which is aimed as an aerotropolis - a means for placemaking.
\end{abstract}

Key words: Clogged water, dual (plural) surveillance, environmental mitigation, MIMO, smart growth.

\section{Introduction}

Although traditionally, negative concerns may be aroused when people face the operated surveillance, the aggressive surveillance (typically with cameras) has become empathetically required since more than two decades ago, e.g., in casinos and theme parks. To positively concern terrorism, and other unexpected incidents or challenges, the demand of AI (artificial intelligence) or biologic plural surveillance, such as two (dual) eyes or two ears and/or the combination of different senses_-including, exemplarily the olfactory one-can be evidenced from a recent bus-fire disaster happened on Taiwan's freeway in last year (refer to "2016 Taoyuan bus fire" on Wikipedia or other public media).

This accident can be more serious if it happens within a long tunnel; nevertheless, even if in the asphalt pavement or other less qualified fire-resistance environment. Such concerns still may not be well acknowledged by professionals who claim the

Corresponding author: Li-Yen Hsu, Ph.D., head architect-HSU Studio; research fields: spatial information networking, and urbanism. E-mail: liyenhsu@ms19.hinet.net. unexpected conditions and/or quantity forecasts should be well presented, or be operated as the research methodology shown on books, before physical facilities' design or building-up. Actually, spatial safety especially that is related to human behaviors (not purely caused due to natural disasters), cannot be guaranteed by previous conditions. More effectively, efficient capabilities on prevention or mitigation should be concerned before disasters really happen. However, they often seem to need a significant loss of life before things are changed, e.g., referring to "Grenfell Tower fire" happened on June 14, 2017 through Wikipedia. ${ }^{1}$ Such capabilities, related to counter wicked problems or difficultly anticipated problems, are recommended to be designed with integrative, and public welfare oriented prototypes [1].

Such surveillance prototypes can be combined with other information services, including the ERP (electronic road pricing ) system, with radio frequency

\footnotetext{
${ }^{1}$ Two fire disaster cases for proactive reviewing on Wikipedia: https://en.wikipedia.org/wiki/2016_Taoyuan_bus_fire; https:// en.wikipedia.org/wiki/Grenfell_Tower_fire.
} 
identification and cameras [2], along the road which has been successfully promoted by Singapore since last century. Such wireless communication related mechanism can be classified as a part of the dedicated short range communications, which is contemporarily considered as an element in the ITS (intelligent transport systems). On building trust in global cooperation business and tourism, dealing with any incidents, environmental issues should be seriously taken care of. From Singapore's ERP experience, designing more reliable (wireless communication) surveillance can benefit developing ITS and sustainable resource utilizations. In this century, aerotropolis development is essential for global economies; resilient airport cooperation and/or synergic benefits should be deeply considered [3], e.g., ferry ports and shoreline parks can be connected and managed together-heavily via wireless control now.

Radio communication integrity deeply counts on the fault-tolerance and the capabilities countering environmental challenges including humidity, dynamic occlusion, radio interference, or jamming. To help smart urban growth as well as to well inform the government, media, travellers, and residents, enhancing spatial connectivity and flexibility also should be well designed with the ITS. Then the availability of evidence can help profile (globally concerned) events in time. Furthermore, the systematic maintenance inspection, including the security mattered, is to be frequently operated through reasonable order, such as the mathematical Hamiltonian properties of proposed prototypes.

Such an infrastructure is prototyped to use the mathematical, optimal in-terms-of dual surveillance based regular degree-three SW (spider-web network) to be placed, with such as gantries, along connected critical passages, and the regular degree-three CCC (cube-connected cycle), with such as probes, for sea, wetlands and sparsely populated areas. Its configuration adaptability can be planned to offer integrated resilience. The approach of establishing free-interference concerned dedicated short range communications [4] for similar inherent conditions of the July 19, 2016 Taoyuan bus fire event has already gotten the consensus on establishing wireless remote surveillance, ${ }^{2}$ which also can justify that a reliable networking prototype should be proactively studied or strategically designed. In fact, many bus companies in Taiwan had operationally installed several cameras and/or other sensors on the bus' interior and exterior for several years. Nevertheless, especially the olfactory biosensors can help detect the volatile chemicals of this fire disaster.

In summary, wireless plural surveillance prototype is inherited with availability, reliability, maintainability, and information integrity (such as the ability to counter interference and to send dynamic secret information) in spatial information network infrastructure and can support safety and security, e.g., in the following applications: (1) to counter international terrorism; (2) to concern radio interference due to humid climate and other environmental issues; (3) to promote knowledge economics and/or international business.

\section{Method (AI Oriented Prototyping)}

Communication/information networks are usually illustrated by graphs in which nodes represent processors and edges represent links between processors. It is noted that mathematically, scalable performance is beneficial in building up a network prototype; the scalability is also important for establishing a communication/information sensor-node platform to flexibly support offering the availability for dealing different environment conditions; the mathematical Hamiltonian order helps guarantee maintenance justifiably done (without loss, and with rational efficiency). This paper proposes an approach on the reliability in establishing communication/information networks for managing,

\footnotetext{
${ }^{2}$ https://udn.com/ news/story/10066/1953785.
} 
serving areas which require quite significant amount of sensor-nodes for reliable communication/information acquiring, serving and managing.

Let $G=(V, E)$ be a graph if $V$ is a finite set and $E$ is a subset of $\{(a, b) \mid(a, b)$ is an unordered pair of $V\}$. A path is delimited by $\left(x_{0}, x_{1}, x_{2}, \ldots x_{n-1}\right)$. A path is called a Hamiltonian path if its nodes are distinct and span $V$. A cycle is a path of at least three nodes such that the first node is the same as the last node. A cycle is called Hamiltonian cycle or Hamiltonian if its nodes are distinct except for the first node and the last node, and if they span $V$.

A bipartite graph $G=(V, E)$ is a graph such that $V=$ $A \cup B$ and $E$ is a subset of $\{(a, b) \mid a \in A$ and $b \in B\}$; if $G-F$ remains Hamiltonian for any $F=\{a, b\}$ with $a$ $\in A$ and $b \in B$, then $G$ is $1_{p}$-Hamiltonian. A graph $G$ is 1-edge Hamiltonian if $G-e$ is Hamiltonian for any $e \in E$; moreover, if there is a Hamiltonian path between any pair of nodes $\{c, d\}$ with $c \in A$ and $d \in$ $B$, then the bipartite graph $G$ is Hamiltonian laceable. It is noted that laceability is used for concerning the connectivity to keep extended areas being integrated, or vice versa, an area can be managed hierarchically yet effectively.

The bipartite spider web network, $\operatorname{SW}(m, n)$, is the graph with the node set $\{(i, j) \mid 0 \leq i<n\}$, where $m$ and $n$ are $\geq 4$, even integers such that $(i, j)$ and $(k, l)$ are adjacent if they satisfy one of the following conditions: (1) $i=k$ and $j=l \pm 1$; (2) $j=l$ and $k=i+1(\bmod m)$ if $i+j$ is odd or $j=n-1$; (3) $j=1, k=i-1(\bmod m)$ if $i$ $+j$ is even or $j=0 . \mathrm{SW}(m, n)$ is proved to be $1-$ edge Hamiltonian and $1_{p}-$ Hamiltonian [5]. Thus, the fault-tolerance involved is systematically based, i.e., prototyped to capably deal with the unexpected incident at any time and at any location, even at several locations concurrently. Moreover, $\operatorname{SW}(m, n)$ is Hamiltonian laceable [6]. The number of links connecting a node is called the degree, and networks regularly having smaller degree are economic in general [7]. Two Hamiltonian paths, $P_{1}=\left(u_{1}, u_{2}, \ldots\right.$,
$\left.u_{n}(G)\right)$ and $P_{2}=\left(v_{1}, v_{2}, \ldots, v_{n}(G)\right)$ of $G$ from $u$ to $v$ are independent if $u=u_{1}=v_{1}, v=u_{n}(G)=v_{n}(G)$, and $u_{i} \neq$ $v_{i}$ for every $1<i<n(G)$. A set of Hamiltonian paths, $\left\{P_{1}, P_{2}, \ldots, P_{k}\right\}$, of $G$ from $u$ to $v$, are mutually independent if any two distinct paths in the set are independent from $u$ to $v$ [8]. It was found that $\mathrm{SW}(m$, $n$ ) has the performance of at least two MIHPs (mutually independent Hamiltonian paths) between any pair of bipartite nodes [9]. It is noted that MIHP is considered for parallel, pact wireless information transmission, diagnosing, and offering additional ciphered information, which is considered important for offering real-time private information to logistic consigner.

The definition of the hypercube is given as follows. Let $u=b_{n-1}, \ldots, b_{i}, \ldots, b_{0}$ be an $n$-bit binary string. For any $j, 0 \leq j \leq n-1$, we use $(u)_{j}$ to denote the binary string $b_{n-1}, \ldots, b_{i}, \ldots, b_{0}$. Moreover, we use $(u)_{j}$ to denote the bit $b_{j}$ of $u$. The Hamming weight of $u$, denoted by $\mathrm{wH}(u)$, is the value of $\mid\left\{0 \leq i \leq n-1 \mid(u)_{i}=\right.$ $1\} \mid$. The hypercube $Q_{n}$ consists of $2^{n}$ nodes and $n 2^{n-1}$ links. Each node corresponds to an $n$-bit binary string. Two nodes, $u$ and $v$, are adjacent if and only if $v=(u)_{j}$ for some $j$, and we call link $\left(u,(u)_{j}\right) j$-dimensional. The Hamming distance between $u$ and $v$, denoted by $h(u, v)$, is defined to be the number of elements in $\{0$ $\left.\leq i \leq n-1 \mid(u)_{i} \neq(v)_{i}\right\}$. Hence, two nodes, $u$ and $v$, are adjacent if and only if $h(u, v)=1$.

The cube-connected-cycles graph $\mathrm{CCC}_{n}$ has $n 2^{n}$ nodes labeled as $(l, \boldsymbol{x})$, where $l$ is an integer between 0 and $n-1$ and $\boldsymbol{x}$ is a processor node with an $n$-bit binary string. Two vertices $(l, \boldsymbol{x})$ and $(l, \boldsymbol{y})$ are adjacent if and only if $\boldsymbol{x}=\boldsymbol{y}$ and $\left|l-l^{\prime}\right|=1$ or $l=l^{\prime}$ and $\boldsymbol{y}=(\boldsymbol{x})^{l}$. In the final condition, $\boldsymbol{x}$ differs with $\boldsymbol{y}$ only in the bit positioned at $l$. The edges that connect $(l, x)$ to its neighbors $(l+1, \boldsymbol{x})$ and $(l-1, \boldsymbol{x})$ are called cycle edges. Moreover, these cycle edges form a cycle of length $n$, which is called a fundamental cycle and is defined by $\boldsymbol{x}$. For $n=2, \mathrm{CCC}_{n}$ is simply a cycle graph of length 8 . Furthermore, $L(n)$ is the set of all possible lengths of the cycles in $\mathrm{CCC}_{n}[10,11]$. 


\section{Features}

Regarding the suitability of research methodology, "2016 Taoyuan bus fire" justifies that the prototyped plural-surveillance approach in dedicated short range communications of the ITS is needed; similar to people's using two (dual) eyes, two ears, and other sensory, a real-time reliable surveillance need such (creature) mechanism, i.e., availability, to count blind spots, to distinguish a little difference, to confirm messages, and/or to concern node fault-tolerance, and node reliability. In terms of prototyping systematic availability, the methodology of this research can be described as design or task based.

Effective maintainability of a relative large amount of nodes especially on those security related ones should concern the sequential inspection without loss and repetition; such operation is the proven mathematical Hamiltonian. Hamiltonian also can support some edge fault-tolerance (reliability); more fault-tolerance can be supported through 1 - edge Hamiltonian and/or $1 p$ - Hamiltonian, which can casually help the network element's repairing (different from the need of the probe application). Proven laceability of the bipartite spider-web network helps establish network hierarchies and the aerial connectivity, or spatial flexibility in promoting smart urban growth. The bipartite spider-web network naturally is dual-node prototype based; however, it also can be adapted, specifically, e.g., to merge sub-nodes. The cube-connected cycle is considered for the probe application; it can support availability on both terms-Hamiltonian and plural surveillance. Prototyped mathematic models can also support scalability, which helps accommodate different conditions, such as the path width and the traffic volume.

National developments should not compromise needs of future generations in creating goods [12]. Consequently, providing sensitive (plural or dual surveillance as the method for problem solving) and reliable information-service infrastructures for place security and development is needed. They naturally need holistic observations in trustworthy ways (i.e., availability, maintainability, reliability) to counter environmental changing, disasters, and any other unexpected events. Especially, intentional crimes or terrorism activities should be counted, though, mostly from the sightline perspectives since last century.

From the lesson of "2016 Taoyuan bus fire", the VANET (vehicular ad-hoc network in ITS) is not almighty because the purely private vehicles cannot be offered a governing duty to guarantee their capabilities. Further surveillance control technologies including those for promoting radio information integrity are needed along paths, especially for managing radio-advanced unmanned aerial vehicles. Developing MIHP, which naturally needs financial support, was aimed before MIMO (multi-input multi-output) or other technologies available for counting radio interference; yet such MIHP performance still can benefit the privacy protection from the coding through the active radio frequency identification (Fig. 1).

\section{Frequently maintenance inspection (flexibly) through the mutually independent Hamiltonian paths} help comparatively analyze interference $(\mathcal{N})$, and flexibly offer a dynamic cipher coding mechanism.

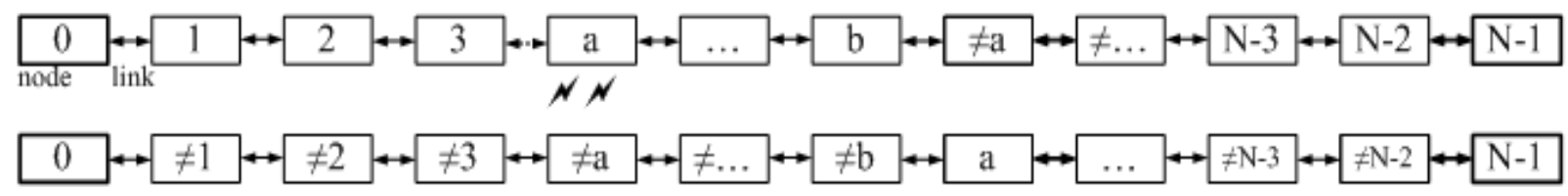

Fig. 1 Diagnose interference and assign cipher codes via "mutually independent Hamoltonian path" MIHP. 


\section{Shoreline Promotion}

Global climate changes, including those causing the rise of the sea level, are apparently threatening the living environment. For environmental mitigation, Taiwan's coast and its outlying islands should have reliable, incorporative area-based security-information networks to protect their environments and security, which is also related to living. It is also suggested to promote the land-use along the shoreline including the built-up of luxury hotels, tourism resorts.

Furthermore, the reclamation of coast lands can cause to create transportation, logistic infrastructures including ports and airports. Modern technologies, developed on existing issues [10, 11], can be rationally counted to help control natural challenges and to promote human well-beings, due to their quality level on reliability engineering.

Speedy ferries with safe wind resistance capabilities for utilizing neighboring seas and with relatively small amount of investment, can offer working opportunities, i.e., social equity, for ordinary citizens. In terms of economic scale, such speedy ferries, similar to container trucks on roads, can be efficiently used instead of through air freight across the Taiwan Strait, such as from Hsinchu, Taiwan to Xiamen, Fujian, mainland China, especially if such speedy ferries (vehicles) can be used on both waterways and roads. Safety and security along waterways is naturally important, and radio integrity related to the humidity factor is considered in the proposed networking. Furthermore, smuggling control naturally could have been considered.

Moreover, integrative tourism development with the sea is very meaningful, practical in Taiwan, due to the following reasons: (1) Formosa (the beautiful island) is another name of Taiwan due to its powerful and/or beautiful hilly sceneries viewed from the sea; (2) many resources can be developed from the sea nowadays, and Taiwan closes to important international water transportation routes; (3) historical settlements of Taiwan started from ports, (not from the road) and can be tourism development assets; (4) Taiwan's airport development counts on open water areas to take advantages of shipping logistics and the relative safety factor in airplane landing and departing.

\section{Space with Care and Integrity}

Contemporary urbanism should comprehensively consider both historical values and future needs. Historical or original assets still can be used if their performance can be improved through adaptation. That is not only for resource saving but also for creating more wonderful spatial performance (including tourism development) or for maintaining society harmony. Besides, social equity such as creating employment opportunities for people eager to earn a basic living (such as the job of landscaping, vehicle drivers) is worthwhile to be considered.

Aerotropolis development should count airport resilience. However, due to noise, safety, and other environmental reasons, Taiwan's first international airport, Songshan airport, which can offer air transport resilience to the northern Taiwan, has been suggested to be moved outside Taipei city recently. Two factors are tentatively established here: (1) advanced technologies, such as integrative surveillance systems, can benefit free aviation, increase transport safety, and lessen adversary environmental impacts; (2) well developing open water areas have opportunities to promote tourism via ships and to benefit gaining both the financial acquisition and the citizen participation in protecting the shoreline. Moreover, such water areas are also leisure resources for domestic people.

Including in Taiwan, unless land acquisition benefits or payments can be satisfied by the landowners, social unrests can easily be aroused even planning to build new airport facilities proclaimed for public interests. Besides, transforming the original airport to the new site may create new problems and potentially cannot avoid same problems, like noise 
and limited safe airspace; i.e., happening again if referring to original airport site's development history. Hence, modifying the original airport by utilizing higher technologies and positive management can be a relatively easier approach.

Green recreation spaces, intended for increasing Taipei city residents' welfare, via moving out the existing airport and its related land-use, had been intuitively proposed. Yet, Louvre pyramid, which had been designed by Architect I. M. Pei to keep the historical asset museum without any loss, and even can well gain demanded spaces and increase the integral site quality by means of applying critical capabilities, such as the high performance, transparent glass [13]. Similarly, with the proposed security-information capabilities (in terms of better aviation operation, general safety, and customer services) and the water recreation facilities on the neighboring river, the value of the existing airport can be highlighted (Fig. 2).

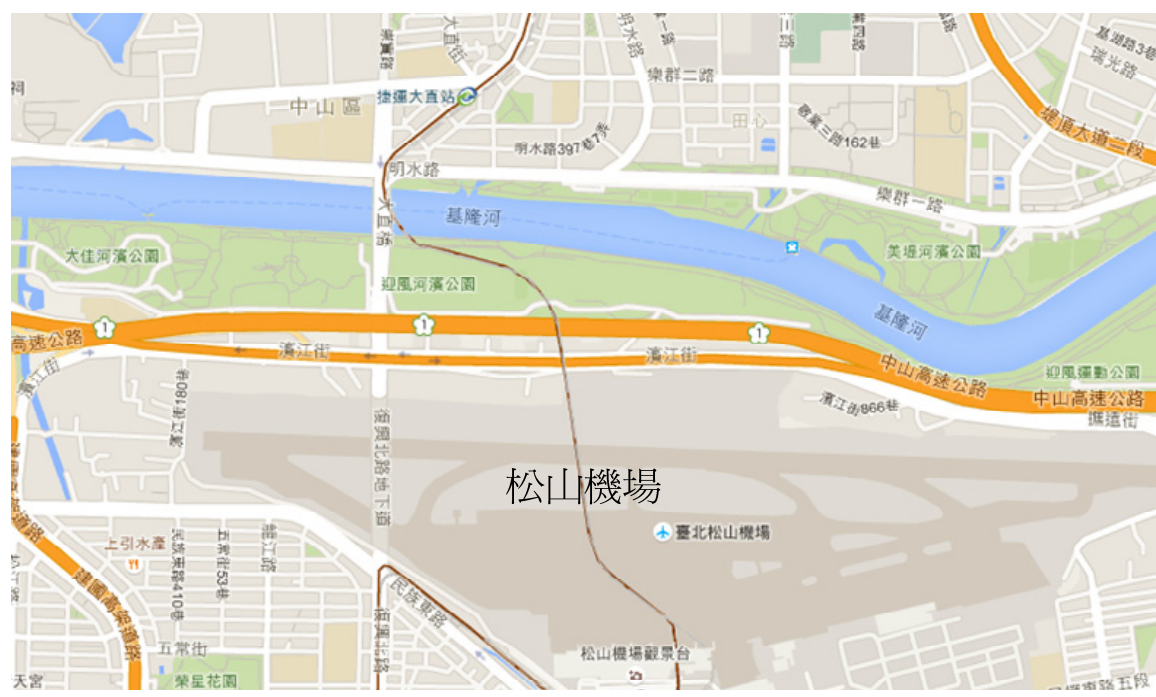

(a)

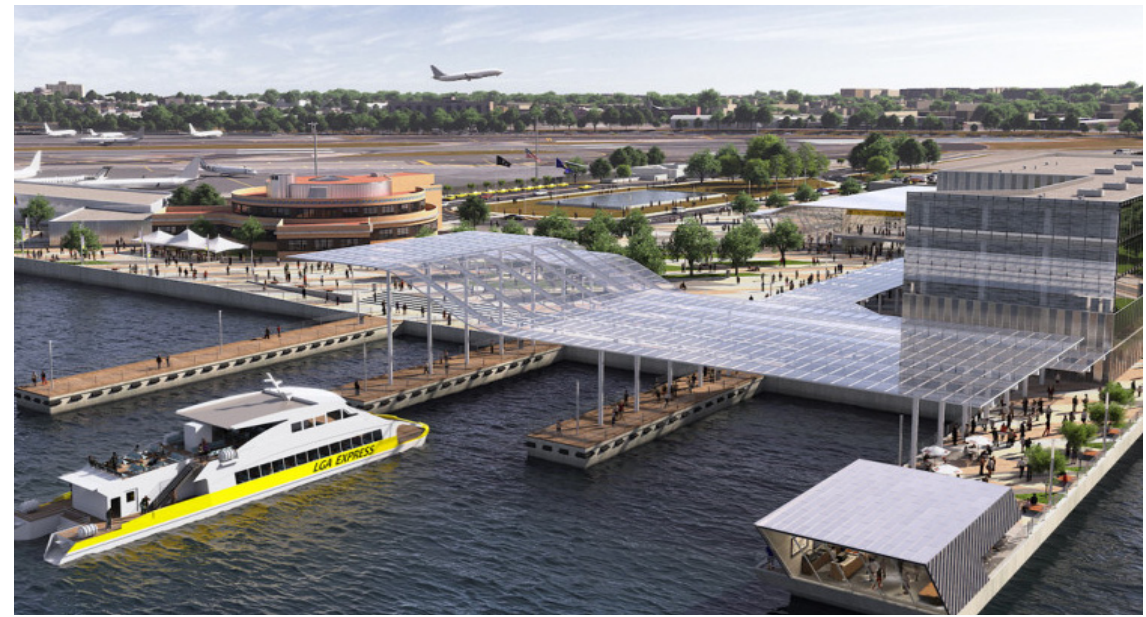

http://www.architectmagazine.com/people/sashaboglu

(b)

Fig. 2 Songshan airport, with a ferry terminal, proposed as an element of Taiwan aerotropolis: (a) it is along the Keelung river; (b) the earliest airport of New York, LaGuardia airport, can be its reference. 


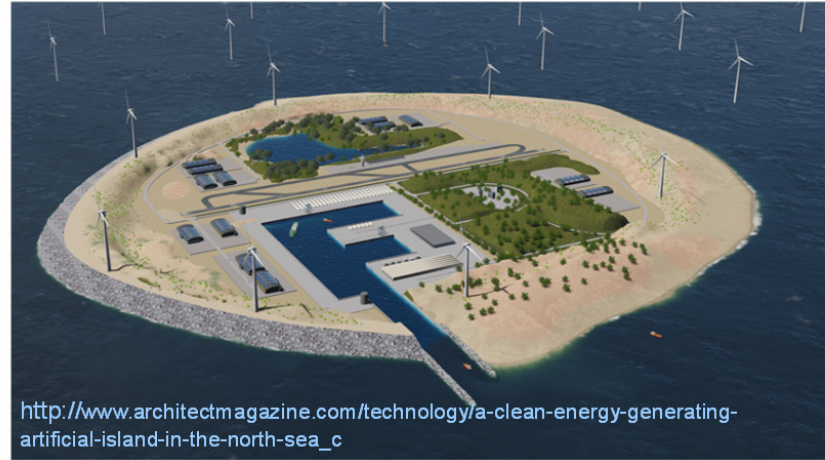

(a)

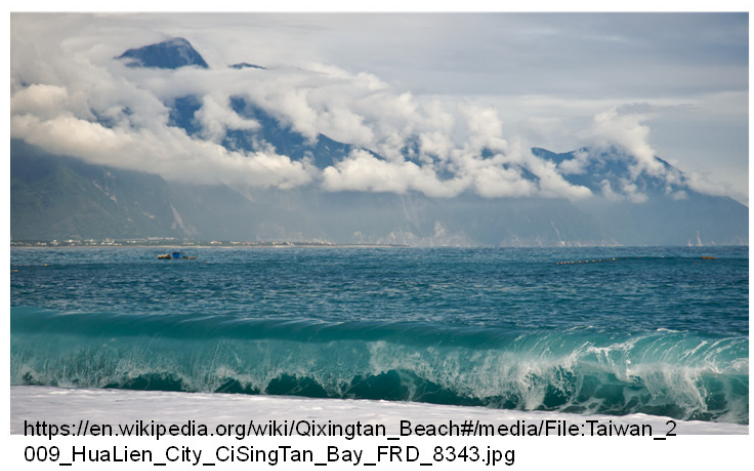

(b)

Fig. 3 Coastline engineering needs promote original values and future welfare: (a) a newly established artificial island supporting international welfare; (b) a view of the east coastline of Taiwan and the image of Formosa-beautiful island with sea and mountain.

Source: Ministry of Transportation and Communications.

Shoreline protection naturally can be related to the tourism development via sea and ports of Taiwan. Then, improving shoreline views needs to be integrally and carefully considered. Specifically, those hilly coast areas, which often have landslide related issues, can be integrally planned to improve the view from the sea, land-use safety, providing soil resource for coast land reclamation (Fig. 3). In this era of global changing, the principle of carefully creating resource should be followed; consequently, plural surveillance based areal surveillance-information should be studied.

\section{Conclusion}

Trust building or civic participation in placemaking needs to get more attention from the authority. Developments should not compromise others' rational benefits. Flexibility in urban planning is more rationally anticipated; that "do not blindly believe forecasts" and "humanistic marketing promotes placemaking" is assumed.

Just as mankind use two eyes for seeing, two ears for hearing, two nostrils for smell, to well sense and communicate the changing environments and related information, plural (dual) surveillance-information networking is suggested, especially in the era that wireless telecommunications are naturally prevailed.
The capital of Taiwan is Taipei, a world city. In other words, Taiwan's economy significantly counts on globalization activities; just-in-time economy can push the strategy of using the speedy ferry and some unmanned aerial vehicles, instead of freight aviation across the strait. Taiwan's geo-political economy can help it being as a successful aerotropolis, which naturally accommodates tourism and business if security-information services can be synergistically acknowledged. Consequently, aiming Taiwan to be an aerotropolis of ports and airports is a rational task, for achieving it, a problem-solving oriented, performance mathematically evidenced research being prototyped to be an intelligent transportation subsystem here.

\section{Acknowledgments}

The author would like to thank the support of ucplyh001 on the utilization of Ansys product HFSS from National Center for High Performance Computing, and encouragements from colleagues, including those in the 2017 international conference on civil and building informatics (ICCBEI).

\section{References}

[1] Fisher, T. 2014. "Public Values and the Integrative Mind: How Multiple Scores Can Collaborate the City Building." Public Administration Review 76 (4): 457-64.

[2] Gómez-Ibáñez, J., and Small, K. 1994. Road Pricing for Congestion Management: A Survey of International 
Practice. Washington D.C.: Transportation Research Board, National Research Council, National Academy Press.

[3] Ashford, N., Norman, J., Mumayiz S., and Wright, P. 2011. Airport Engineering: Planning, Design, and Development of 21st Century Airports. New York, NY: John Wiley, 673-81.

[4] Anderson, J., Kalra, N., Stanley, K., Sorensen, P., Samaras, C., and Oluwatola, O. 2016. Autonomous Vehicle Technology-A Guide for Policymakers. Santa Monica, CA: RAND Corporation, 71-80. Accessed July 21, 2017. http://www.rand.org/pubs/research_reports/ RR443-2.pdf.

[5] Kao, S.-S., and Hsu, L.-H. 2005a. "Spider Web Networks: A Family of Optimal, Fault Tolerant, Hamiltonian Bipartite Graphs." Applied Mathematics and Computation 160 (1): 269-82.

[6] Kao, S.-S., and Hsu, L.-H. 2005b. "Hamiltonian Laceability of Spider Web Networks." Chung Yuan Journal (Taiwan) 33 (1): 1-10.

[7] Stojmenovic, I. 1997. "Honeycomb Networks: Topological Properties and Communication Algorithms."
IEEE T PARALL DISTR 8 (10): 1036-42.

[8] Teng, Y.-H., Tan, J., Ho, T.-Y., and Hsu, L.-H. 2006. "On Mutually Independent Hamiltonian Paths." APPL MATH LETT 19 (4): 345-50.

[9] Hsu, L.-Y. 2012. "Scalable Parallelism in Spider-Web Networks." Int'l J. Information Engineering 2 (4): 169-83.

[10] Hsu, L.-Y. 2016a. "Configuring Availability, Reliability, Maintainability-A Taiwan's Air-Sea Port as an Example." J. Communication Technologies \& Research 4 (1): 4-12. Accessed July 21, 2017. https://www.erpublication.org/admin/vol_issue2/upload\% 20Image/IJMCTR041105.pdf.

[11] Hsu, L.-Y. 2016b. "Sustainable Placemaking at Taiwan's Shoreline-Thinking from Hsinchu/The Shortest Path across the Taiwan Strait." In Proceedings of International Chinese Transportation Professional Association (ICTPA), May 28, Hsinchu, ASCE, 78-85.

[12] Orsato, R. 2016. Sustainability Strategies: When Does It Pay to Be Green?. New York, NY: Palgrave, 4.

[13] Mercier, D. 2017. "The Ancien Régime's Fatal Blow.” $J$. AIA. July, 64-6. 